\title{
COMPORTAMIENTO AGRONÓMICO DE HÍBRIDOS Y PROGENITORES DE SORGO PARA GRANO EN LAS HUASTECAS ${ }^{1}$
}

\author{
Héctor Williams-Alanís ${ }^{2}$, Gerardo Arcos-Cavazos ${ }^{3}$
}

\begin{abstract}
RESUMEN
Comportamiento agronómico de híbridos y progenitores de sorgo para grano en las Huastecas. El objetivo de este estudio fue medir la productividad de un grupo de híbridos experimentales de sorgo para grano en la región de las Huastecas, contrastándolos con los testigos comerciales y progenitores. Durante los ciclos primavera verano 2005, 2006 y 2007 en temporal, se establecieron en Estación Cuauhtémoc, Tamaulipas, México, tres ensayos con 49 híbridos experimentales y comerciales. En el 2008 durante el mismo ciclo en temporal, se estableció un ensayo con 28 híbridos y progenitores. Se utilizó un diseño de bloques completos al azar con cuatro repeticiones. Se identificaron híbridos experimentales agronómicamente similares a los comerciales. Uno de los mejores fue RB-104xRTx437, el cual produjo $6403 \mathrm{~kg} / \mathrm{ha}$ de grano. Fue igual $(\mathrm{p} \leq 0,05)$ a: RB-Patrón $(6288 \mathrm{~kg} / \mathrm{ha}), \mathrm{A}$. Ámbar $(6028 \mathrm{~kg} / \mathrm{ha}), \mathrm{DK}-65$ (5763 kg/ha) y RB-4000 (5700 kg/ha), y superior a Pioneer $82 \mathrm{G} 63$ (4796 kg/ha), Pioneer 84G62 (4256 kg/ha) y Z-400 $(4218 \mathrm{~kg} / \mathrm{ha})$. Este híbrido fue $(\mathrm{p} \leq 0,05)$ más precoz a la floración (62) que A. Ámbar (65) y DK-65 (66). Los híbridos experimentales fueron más rendidores $(2924 \mathrm{~kg} / \mathrm{ha})$, más altos $(17 \mathrm{~cm})$, más precoces $(-4,5 \mathrm{DF})$, de mayor longitud de la espiga $(0,9 \mathrm{~cm})$ y exserción $(3,5 \mathrm{~cm})$, y menor incidencia de enfermedades foliares $(-0,24)$ que sus padres. RTx 437 , fue el mejor progenitor con un rendimiento de grano $(3598 \mathrm{~kg} /$ ha) igual $(\mathrm{p} \leq 0,05)$ a los híbridos.
\end{abstract}

Palabras clave: Sorghum bicolor, mejoramiento genético de plantas, rendimiento de grano, heterosis, heterobeltiosis.

\begin{abstract}
Agronomic performance of hybrids and parents of grain sorghum in Huastecas. The objective of the study was to determine the productivity of experimental hybrids of grain sorghum group in the Huastecas region, contrasting with commercial checks and parents. At Estación Cuauhtémoc, Tamaulipas, Mexico, three trials with 49 experimental and commercial hybrids were established during the springsummer cycles 2005, 2006 y 2007 in dry land conditions. In 2008 in dry land, a trail was established with 28 hybrids and parents. In all cases we used a randomized block design with four replications. Experimental hybrids were identified as agronomically similar to commercials. One of the best hybrids was RB-104xRTx437, which produced $6403 \mathrm{~kg}$ / ha of grain. It was equal $(\mathrm{p} \leq 0.05)$ to: RB-Patron $(6288 \mathrm{~kg} /$ ha), A. Amber (6028 kg/ha), DK-65 (5763 kg/ha) and RB$4000(5700 \mathrm{~kg} / \mathrm{ha})$ was superior to Pioneer 82G63 (4796 $\mathrm{kg} / \mathrm{ha})$, Pioneer 84G62 (4256 kg/ha) and Z-400 (4218 kg/ ha). The hybrid was also $(\mathrm{p} \leq 0.05)$ the earliest to flower $(62$ d) compared to to A. Ambar (65 d) and DK-65 (66 d). The experimental hybrids were higher yielding (2924 kg/ha), taller $(17 \mathrm{~cm})$, more precocious $(-4.5 \mathrm{DF})$, had longer panicle length $(0.9 \mathrm{~cm})$ and exsercion $(3,5 \mathrm{~cm})$, and lower foliar diseases incidence $(-0.24)$ than their parents. RTx437 was the best parent with grain yield $(3598 \mathrm{~kg} / \mathrm{ha})$ equal $(\mathrm{p} \leq 0.05)$ to the hybrids.
\end{abstract}

Keywords: Sorghum bicolor, plant breeding, grain yield, heterosis, heterobeltiosis.

\footnotetext{
Recibido: 10 de abril, 2014. Aceptado: 9 de setiembre, 2014. Proyecto de investigación No. 2016247A financiado por la Fundación Produce Tamaulipas, A.C., México.

2 Universidad Autónoma de Nuevo León, Facultad de Agronomía (FAUANL), km 17.5 Carr. Zuazua-Marín, Marín, Nuevo León. México. 66700. hectorwilliamsa@yahoo.com.mx (Autor para correspondencia).

3 Instituto Nacional de Investigaciones Forestales, Agrícolas y Pecuarias (INIFAP). Campo Experimental Huastecas. Carr. Tampico-Cd. Mante Km. 55 Apdo. Postal C-1 Suc Aeropuerto, Tampico, Tam., México. CP 89339. arcos.gerardo@inifap.gob.mx
} 


\section{INTRODUCCIÓN}

En México, el sorgo [Sorghum bicolor (L.) Moench] representa el segundo cultivo de mayor importancia en cuanto a producción de grano. La superficie de siembra para esta gramínea fluctúa alrededor de dos millones de hectáreas, de las cuales se obtiene una producción promedio anual de seis millones de toneladas (Financiera Rural, 2011a). En la región de las Huastecas, que comprende áreas agroclimáticas similares, que incluyen el sur de Tamaulipas, oriente de San Luís Potosí y norte de Veracruz, se cultiva una superficie de $180 \mathrm{mil}$ hectáreas de sorgo para grano al año y se obtiene una producción cercana a 400 mil toneladas (SIAP, 2011). Sin embargo, se presenta el problema de la escasez de híbridos y variedades de sorgo (S. bicolor) para grano, para condiciones de riego y temporal; con buena producción de grano y tolerantes a enfermedades; además la semilla para siembra es importada y tiene un alto costo (Williams et al., 2006). De acuerdo con cifras de la Secretaría de Economía, México importó en 2010, 36400 toneladas de semilla de sorgo, con un valor de 43,8 millones de dólares. Esta semilla se utilizó para la plantación de dos millones de hectáreas del cultivo (Financiera Rural, 2011b). México importa prácticamente toda la semilla que utiliza para siembra. Esto genera dependencia tecnológica, incremento de los costos de producción, reducción de la rentabilidad y disminución de la competitividad de los productores (CONACYT, 2013).

El descubrimiento de la androesterilidad genética citoplásmica por Stephens y Holland (1954), hizo posible la producción comercial de semilla híbrida. Los primeros híbridos desarrollados en EU se empezaron a utilizar en México a partir de 1958 (Williams et al., 2006). A la fecha casi el $100 \%$ del sorgo cultivado es de semilla híbrida, donde se aprovecha su mayor potencial de producción (Flores-Naveda et al., 2013).

En Estados Unidos se estima que, al empleo de los híbridos de sorgo, se les atribuye entre el 35\% y $40 \%$ de la ganancia obtenida en producción de grano; mientras que a las prácticas culturales se asigna del 60 al 65\% del incremento (Duvick, 1999). En Kansas EUA, se estima que mediante el mejoramiento genético de sorgo en temporal, en 51 años (1957-2008); el potencial de rendimiento de grano se incrementó en $1430 \mathrm{~kg} / \mathrm{ha}$, lo que equivale a un $139 \%$ de aumento
(Assefa y Staggenborgh, 2009). En México en el Campo Experimental Río Bravo (CERIB) del Instituto Nacional de Investigaciones Forestales, Agrícolas y Pecuarias (INIFAP); se estima que el avance obtenido en mejoramiento genético en un periodo de once años para condiciones de riego y buen temporal, fue de $1100 \mathrm{~kg} / \mathrm{ha}$ (Williams et al., 1995).

La investigación en mejoramiento genético de sorgo en el norte de México, por parte del Instituto Nacional de Investigaciones Agrícolas, INIA (hoy INIFAP); se inició en el Campo Experimental Río Bravo en 1974. Su ámbito incluyó las regiones cálido-secas del país en los estados de Tamaulipas, Nuevo León, Coahuila, Chihuahua, Sonora y Sinaloa (Williams et al., 1995).

Los materiales generados en INIFAP, Río Bravo; RB-3030, RB-3006, RB-4000, RB-4040 y RB-Patrón, presentaron adaptación en la región de las Huastecas. (Williams, 1981; Williams, 1996; Williams et al., 2004). RB-3030 desarrollado en 1980, presentó un potencial de producción de grano en el noreste de México $16,9 \%$ superior a los híbridos comerciales evaluados (Williams, 1981). Por otro lado, RB-4000 formado en 1987 (Williams, 1988), presentó un rendimiento de grano experimental de $14 \%$ más respecto los mejores híbridos comerciales (Williams, 1988). En el año 2003 se registró el híbrido RB-Patrón, el cual presentó características fisiológicas, morfológicas y bioquímicas que le confieren tolerancia al estrés producido por la sequía y altas temperaturas (Williams et al., 2004). Este híbrido al ser evaluado en la región de las Huastecas en parcelas de validación con productores, presentó rendimientos de grano superiores en más de $20 \%$ comparado con los híbridos comerciales.

Elobjetivode este estudio fuemedirla productividad de un grupo de híbridos experimentales de sorgo para grano en la región de las Huastecas, contrastándolos con los testigos comerciales y progenitores.

\section{MATERIALES Y MÉTODOS}

Se evaluaron 33 híbridos experimentales pertenecientesalINIFAP; cincohíbridosexperimentales de la Facultad de Agronomía de la Universidad Autónoma de Nuevo León (FAUANL) y once híbridos comerciales (Cuadro 1). Los híbridos experimentales fueron seleccionados de germoplasma formado en el 
Cuadro 1. Relación de híbridos de sorgo (Sorghum bicolor) evaluados en Estación Cuauhtémoc, Tamaulipas, México, durante los ciclos P-V 2005, 2006 y 2007.

\begin{tabular}{|c|c|c|c|}
\hline Número & Híbrido & Número & Híbrido \\
\hline 1 & RB-118 x RTx430 a & 26 & RB-116 x RTx437 \\
\hline 2 & RB-118 x RTx435 a & 27 & RB-116 x $25 \mathrm{CEA}^{\mathrm{a}}$ \\
\hline 3 & $\mathrm{RB}-118 \times 204^{\mathrm{a}}$ & 28 & RB-106 x RTx $430^{\circ}$ \\
\hline 4 & $\mathrm{RB}-118 \times 216^{\mathrm{a}}$ & 29 & RB-106 x RTx 437 \\
\hline 5 & RB-118 x 430 CEA $^{a}$ & 30 & RB-106 x $216^{a}$ \\
\hline 6 & RB-118 x RTx437 a & 31 & RB-106 x 25 CEA \\
\hline 7 & RB-118 x 25 CEA $^{a}$ & 32 & $\mathrm{RB}-64 \times \mathrm{RT} \times 430^{\mathrm{a}}$ \\
\hline 8 & $\begin{array}{l}\text { RB-Patrón (RB-104 } \\
\text { xRTx430) }^{c}\end{array}$ & 33 & RB-64 x RTx437 a \\
\hline 9 & RB-104 x RTx435 a & 34 & $46038 \times 430 \mathrm{CEA}^{\mathrm{b}}$ \\
\hline 10 & RB-104 x 25 CEA $^{a}$ & 35 & $46038 \times \mathrm{RT} \times 430 \mathrm{~b}$ \\
\hline 11 & $\mathrm{RB}-104 \times 216^{\mathrm{a}}$ & 36 & $46038 \times \mathrm{RT} \times 435$ \\
\hline 12 & RB-104 x RTx437 a & 37 & $46038 \times 204$ b \\
\hline 13 & RB-27 x 430 REA $^{\text {a }}$ & 38 & $46038 \times \mathrm{RT} \times 437 \mathrm{~b}$ \\
\hline 14 & RB-27 x RTx435 a & 39 & $\begin{array}{l}\text { RB-3030 (RB-5 x } \\
\text { RTx430) }\end{array}$ \\
\hline 15 & $\mathrm{RB}-27 \times 204^{\mathrm{a}}$ & 40 & $\begin{array}{l}\text { RB-3006 (RB-27x } \\
\text { RTx430) }{ }^{c}\end{array}$ \\
\hline 16 & $\mathrm{RB}-27 \times \mathrm{RT} \times 437^{a}$ & 41 & $\mathrm{RB}-4000^{\mathrm{c}}$ \\
\hline 17 & $\mathrm{RB}-5 \times 204^{\mathrm{a}}$ & 42 & $\mathrm{RB}-4040^{\mathrm{c}}$ \\
\hline 18 & RB-5 x 430 REA $^{a}$ & 43 & RB-64 x RTx435 a \\
\hline 19 & $\mathrm{RB}-5 \times \mathrm{RT} \times 437^{\mathrm{a}}$ & 44 & DK-47 d \\
\hline 20 & RB-119 x RTx430 a & 45 & Asgrow Z-400 d \\
\hline 21 & RB-119 x 25 CEA $^{a}$ & 46 & Pioneer 82G63 ${ }^{\mathrm{d}}$ \\
\hline 22 & RB-119 x RTx435 a & 47 & Pioneer 84G62 d \\
\hline 23 & RB-119 x RTx437 a & 48 & Asgrow Ámbar ${ }^{\mathrm{d}}$ \\
\hline 24 & RB-116 x RTx430 a & 49 & DK-65 ${ }^{\mathrm{d}}$ \\
\hline 25 & RB-116 x RTx435 a & & \\
\hline
\end{tabular}

${ }^{a}$ Híbridos experimentales del INIFAP; ${ }^{\text {b }}$ Híbridos experimentales de la Universidad Autónoma de Nuevo León (FAUANL); c testigos comerciales del INIFAP; ${ }^{\mathrm{d}}$ Testigos comerciales compañías privadas. La nomenclatura de los híbridos experimentales se formó colocando primero la clave que corresponden a las líneas hembra, seguida del signo (x) para después colocar la clave de las líneas restauradoras.

INIFAP, Campo Experimental Bajío, INIFAP, Campo Experimental Río Bravo, Texas A\&M University, ICRISAT, India y por la Universidad Autónoma de Nuevo León. Los híbridos comerciales RB fueron formados en el INIFAP, Campo Experimental Río Bravo (Williams, 1981; Williams, 1996; Williams et al., 2004). Los híbridos de las compañías privadas han sido desarrollados en EUA. Los testigos comerciales fueron seleccionados entre los mejores para rendimiento de grano, con base en los resultados obtenidos en las evaluaciones anuales realizadas por el COTESE (Comité Técnico Estatal de Semillas). El sitio experimental se localizó en la Estación Cuauhtémoc, municipio de Altamira, Tamaulipas, en terrenos del Campo Experimental las Huastecas (INIFAP), a $22^{\circ}$ $33^{\prime} \mathrm{LN} / 98^{\circ} 09^{\prime} \mathrm{LO}$. Presenta un clima $\left(\mathrm{Aw}_{0}\right)$ cálido subhúmedo con lluvias en verano y lluvia invernal de 5-10\% (García, 1988). Con promedio de temperatura media anual de $24,5^{\circ} \mathrm{C}$ y de $842 \mathrm{~mm}$ de precipitación (INIFAP, 2013). Ensayos uniformes se establecieron durante el ciclo primavera verano $(\mathrm{P}-\mathrm{V})$ temporal de los años 2005, 2006 y 2007. En otro experimento sembrado en temporal en P-V 2008, se evaluaron los híbridos experimentales que presentaron mayor rendimiento de grano y sus progenitores.

Los ensayos se establecieron bajo un diseño de bloques completos al azar con cuatro repeticiones. En todos los casos, las parcelas experimentales fueron de un surco de $5 \mathrm{~m}$ de largo y $0,80 \mathrm{~m}$ de separación. Se utilizó una densidad de población de 250000 plantas/ ha. El manejo del cultivo se realizó de acuerdo con las recomendaciones que el INIFAP señala para el cultivo de sorgo en el sur de Tamaulipas. Las variables evaluadas fueron rendimiento de grano $\mathrm{kg} / \mathrm{ha}$ (REN, al $12 \%$ de humedad); días a floración (DF, al 50\% de las plantas), y una semana antes de la madurez fisiológica, se midieron altura de planta (AP, en $\mathrm{cm}$, desde el suelo hasta el ápice de la panoja), longitud de panoja (LP, en $\mathrm{cm}$, de la base al ápice), excersión (EX, en $\mathrm{cm}$, de la lígula de la hoja bandera a la base de la panoja), calificación del aspecto de planta (CP) e incidencia de enfermedades foliares (IEF), las cuales se calificaron en forma visual mediante una escala de 1 al 5. Para calificación de planta: uno, es muy bueno; dos, es bueno; tres, promedio; cuatro, abajo del promedio y cinco, malo. Para enfermedades foliares: uno es completamente sana; dos, de 1 a $10 \%$ de daño; tres, de 11 a $25 \%$ de daño; cuatro, de 26 a $40 \%$ de daño y cinco, más de $41 \%$. La toma de datos están de acuerdo a las recomendaciones sugeridas por House (1985).

Análisis estadístico. El efecto del ambiente en el rendimiento y las características agronómicas se cuantificó mediante un análisis combinado en tres ambientes y los 49 híbridos, utilizando el programa 
Stastistical Analysis Systems Institute (SAS Institute, 1999). La comparación de medias se realizó con la prueba de Tukey $(\mathrm{p} \leq 0,05)$, y se hicieron correlaciones simples de Pearson entre las variables. La heterosis se calculó con la fórmula: Heterosis = media de la F1 - media de los padres en porcentaje con respecto a la media de los progenitores; la heterobeltiosis = Media de la F1 - media del progenitor superior, también en porcentaje con respecto a la media de este progenitor (León-Velasco et al., 2009).

\section{RESULTADOS Y DISCUSIÓN}

\section{Rendimiento de grano}

El análisis de varianza por ambiente indicó diferencias significativas $(p \leq 0,01)$ entre genotipos en los tres ambientes y en el combinado para los tres ambientes (Cuadro 2). Lo anterior es consecuencia de las diferencias genéticas entre genotipos.

También se detectaron diferencias significativas $(\mathrm{p} \leq 0,01)$ entre ambientes y para la interacción genotipoambiente. Esto significa que algunos genotipos se

Cuadro 2. Cuadrados medios y significancia estadística de 49 híbridos de sorgo evaluados en Estación Cuauhtémoc, Tamaulipas, México, durante los ciclos P-V 2005,2006 y 2007.

\begin{tabular}{lcccccccc}
\hline & & \multicolumn{7}{c}{ Cuadrados Medios } \\
\cline { 2 - 8 } Fuente variación & GL & REN & DF & AP & LP & EX & CP & IEF \\
\hline Repeticiones & 3 & $2910792^{* *}$ & $24,4^{* *}$ & $259^{* *}$ & $17,3^{* *}$ & 5,4 & $4,9^{* *}$ & $3,8^{* *}$ \\
Ambientes & 2 & $93072141^{* *}$ & $22125^{* *}$ & $97063^{* *}$ & $48,3^{* *}$ & $6800^{* *}$ & $12,6^{* *}$ & $44,5^{* *}$ \\
Interacción AxR & 6 & $3985088^{* *}$ & $12,2^{* *}$ & $814^{* *}$ & $24,9^{* *}$ & $92,3 * *$ & $1,7^{* *}$ & $2,7 * *$ \\
Variedades & 48 & $6326057^{* *}$ & $74 * *$ & $3266^{* *}$ & $45,8^{* *}$ & $65,9^{* *}$ & $0,5^{*}$ & $0,64 * *$ \\
Interacción RxV & 144 & 866374 & 2,4 & 41 & 2,4 & 6,2 & 0,3 & 0,26 \\
Interacción AxV & 96 & $1692950^{* *}$ & $21,9^{* *}$ & $130^{* *}$ & 4,2 & $12,1^{* *}$ & 0,3 & 0,35 \\
CV \% & & 14,6 & 2,6 & 3,7 & 5,6 & 15,1 & 19,1 & 19,3 \\
\hline
\end{tabular}

* Significativo. ** Altamente significativo. GL= grados de libertad; REN= rendimiento de grano $\mathrm{kg} / \mathrm{ha}$; $\mathrm{DF}=$ días a floración; $\mathrm{AP}=$ altura de planta en $\mathrm{cm} ; \mathrm{LP}=$ longitud de panoja en $\mathrm{cm} ; \mathrm{EX}=$ longitud de excersión en $\mathrm{cm} ; \mathrm{CP}=$ calificación de planta e $\mathrm{IEF}=$ incidencia de enfermedades foliares, $\mathrm{CV}=$ coeficiente de variación.

Cuadro 3. Comparación de medias mediante la prueba de Tukey $(\mathrm{p} \leq 0,05)$ de 49 híbridos de sorgo evaluados en Estación Cuauhtémoc, Tamaulipas, México, durante los ciclos P-V 2005,2006 y 2007.

\begin{tabular}{lccccccc}
\hline Año & $\begin{array}{c}\text { REN } \\
(\mathbf{k g} / \mathbf{h a})\end{array}$ & DF & AP & LP & EX & CP & IEF \\
\hline 2005 & $5802 \mathrm{a}$ & $60,6 \mathrm{~b}$ & $164,7 \mathrm{a}$ & $26,7 \mathrm{a}$ & $22,0 \mathrm{a}$ & $2,9 \mathrm{ab}$ & $2,7 \mathrm{a}$ \\
2006 & $4491 \mathrm{~b}$ & $53,1 \mathrm{c}$ & $126,5 \mathrm{~b}$ & $27,4 \mathrm{a}$ & $11,0 \mathrm{~b}$ & $2,5 \mathrm{~b}$ & $2,0 \mathrm{~b}$ \\
2007 & $5513 \mathrm{a}$ & $68,1 \mathrm{a}$ & $165,3 \mathrm{a}$ & $27,7 \mathrm{a}$ & $12,8 \mathrm{~b}$ & $2,9 \mathrm{a}$ & $2,9 \mathrm{a}$ \\
Promedio & 5269 & 60,6 & 152,2 & 27,3 & 15,3 & 2,8 & 2,5 \\
\hline
\end{tabular}

$\mathrm{REN}=$ rendimiento de grano $\mathrm{kg} / \mathrm{ha} ; \mathrm{DF}=$ días a floración; $\mathrm{AP}=$ altura de planta en $\mathrm{cm} ; \mathrm{LP}=$ longitud de panoja en $\mathrm{cm}$; EX= longitud de excersión en cm; $\mathrm{CP}=$ calificación de planta e $\mathrm{IEF}=$ incidencia de enfermedades foliares. 
comportan de manera diferencial a través de los ambientes. Para rendimiento de grano (Cuadro 3) el año 2005 (5802 kg/ha) fue similar al $2007(5513 \mathrm{~kg} /$ ha) y superiores al 2006 (4491 kg/ha). Las diferencias observadas en rendimiento se atribuyen a la mejor distribución de la lluvia ocurrida durante los años 2006 y 2007 (Cuadro 4), ya que la precipitación total fue similar en todos los ambientes $(484,445 \mathrm{y}$ $557,2 \mathrm{~mm}$ respectivamente). En los tres ambientes y en el promedio de ambientes se encontraron híbridos experimentales que tuvieron un rendimiento similar al de los mejores híbridos comerciales utilizados como testigos (Cuadro 5). En el experimento sembrado en el 2008 (Cuadro 6), en promedio, los híbridos presentaron un rendimiento de grano superior a sus progenitores. Esto coincide con lo reportado por Kirby y Atkins (2000), Kenga et al. (2003) y Mahdy et al. (2011). Entre los progenitores masculinos sobresalen por su capacidad de formar híbridos rendidores: RTx437, RTx430, 25CEA y 430 REA. Los progenitores masculinos que formaron híbridos experimentales con más bajos rendimientos de grano fueron RTx435 y LRB-204. Esto se relaciona con un mayor o menor grado de heterosis en los híbridos.

El material RTx437 fue liberado por su potencial de producción como progenitor para formar híbridos y por su resistencia a enfermedades (Rooney et al., 2003). En este estudio fue el único progenitor que obtuvo un rendimiento de grano $(3598 \mathrm{~kg} / \mathrm{ha})$ igual $(\mathrm{p} \leq 0,05)$ que los híbridos. En sorgo ( $S$. bicolor), Duvick (1999) indicó que el mejoramiento per se de las líneas progenitoras para rendimiento de grano no es tan importante, como su capacidad de formar híbridos con alto grado de heterosis, pues, el contar con padres más rendidores, facilita y hace más económica la producción de semilla de los híbridos. Además, experimentos realizados en el norte de Tamaulipas se encontró que RTx437 fue una de las líneas que formaron los híbridos experimentales más resistentes a carbón de la panoja Sporisorium reilianum (Kühn) Langdon and Fullerton (Williams et al., 2009a) y los más tolerantes a pudrición carbonosa del tallo $M$. phaseolina (Williams et al., 2009b). Siendo estas, las enfermedades más importantes que afectan al cultivo de sorgo en el norte de Tamaulipas. La segunda de ellas afecta al sur del estado y en algunas regiones cuando las condiciones ambientales son adecuadas pueden causar fuertes pérdidas en la producción de grano (Williams et al., 2009b). Se reporta que el mejoramiento de sorgo en cuanto a resistencia a enfermedades y plagas, y al estrés ambiental, es importante para lograr el mayor aumento de la producción de grano (Duvick, 1999; Reddy et al., 2006). RTx437 también es progenitor masculino del híbrido experimental RB-104xRTx437 $(6403 \mathrm{~kg} / \mathrm{ha})$, que sobresalió en los experimentos; donde resultó igual a los testigos comerciales: RBPatrón (6288 kg/ha), A. Ámbar (6028 kg/ha), DK-65 $(5763 \mathrm{~kg} / \mathrm{ha})$ y RB-4000 (5700 kg/ha); y fue superior a Pioneer 82G63 (4796 kg/ha), Pioneer 84G62 (4256 $\mathrm{kg} / \mathrm{ha})$ y Z-400 (4218 kg/ha).

También RB-104xRTx437 presentó buenos resultados en el norte de Tamaulipas (Montes et al., 2014a), lo cual corrobora los resultados obtenidos en este estudio. En México la disponibilidad de semilla de sorgo (S. bicolor) para siembra depende prácticamente de la importada y su costo en el mercado es cada vez más alto (Flores-Naveda et al., 2013). En el año 2010 se liberaron por sus buenas características agronómicas

Cuadro 4. Condiciones climáticas prevalecientes durante el desarrollo del cultivo en Estación Cuauhtémoc, Tamaulipas, México, durante los ciclos P-V 2005, 2006, 2007 y 2008.

\begin{tabular}{lccccc}
\hline Ambiente & $\begin{array}{c}\text { Precipitación } \\
\text { mm }\end{array}$ & Max & Temperatura ${ }^{\circ} \mathbf{C}$ & $\begin{array}{c}\text { Humedad } \\
\text { relativa (\%) }\end{array}$ \\
\cline { 3 - 5 } & 484 & 30,49 & 21,0 & Med & 82,14 \\
$2005(1)$ & 445 & 31,90 & 20,68 & 25,75 & 79,10 \\
$2006(2)$ & 557,2 & 30,4 & 22,57 & 26,29 & 77,72 \\
$2008(3)$ & 400,6 & 24,89 & 14,95 & 26,48 & 81,78 \\
\hline
\end{tabular}

Ambiente 1: del 26 de agosto del 2005 al 10 enero 2006; Ambiente 2: 10 de agosto al 29 de noviembre del 2006; Ambiente 3: del 17 de agosto del 2007 al 4 de enero del 2008 y Ambiente 4: 21 de agosto del 2008 al 18 de enero del 2009. 
Cuadro 5. Comparación de medias mediante la prueba de Tukey $(\mathrm{p} \leq 0,05)$ de híbridos de sorgo evaluados en tres ambientes en Estación Cuauhtémoc, Tamaulipas, México. Ciclos P-V 2005, 2006 y 2007. Mejores cinco, peores cinco híbridos experimentales y testigos comerciales.

\begin{tabular}{|c|c|c|c|c|c|c|c|}
\hline Genotipo & REN (kg/ha) & DF & $\mathbf{A P}(\mathbf{c m})$ & $\mathbf{L P}(\mathbf{c m})$ & $\mathrm{EX}(\mathrm{cm})$ & $\mathbf{C P}$ & IEF \\
\hline RB-104xRTx437 & $6403 \mathrm{a}$ & 62 cdefghi & 161 efgh & 28,8 abcde & 16,7 cdefghi & $2,7 \mathrm{abc}$ & $2,35 \mathrm{abcd}$ \\
\hline RB-119x25 CEA & $6399 \mathrm{a}$ & 65 abcd & $175 \mathrm{bc}$ & $29,7 \mathrm{abcd}$ & $\begin{array}{c}14,0 \\
\text { hijklmno }\end{array}$ & $2,4 \mathrm{c}$ & $2,28 \mathrm{~cd}$ \\
\hline RB-116xRTx430 & $6332 a b$ & 63 bcdef & 163 defg & $30,0 \mathrm{abc}$ & $\begin{array}{c}14,7 \\
\text { efghijklmno }\end{array}$ & $2,5 \mathrm{abc}$ & $2,28 \mathrm{~cd}$ \\
\hline $\begin{array}{l}\text { RB-Patron } \\
(104 x \text { RTx430) (T) }\end{array}$ & $6288 \mathrm{ab}$ & 63 bcdefg & 157 efghik & $30,3 \mathrm{a}$ & $12,5 \mathrm{jklmno}$ & $2,8 \mathrm{abc}$ & $2,58 \mathrm{abcc}$ \\
\hline RB-5xRTx430 REA & $6190 \mathrm{abc}$ & 58 mnopqr & $198 \mathrm{a}$ & $25,4 \mathrm{klmn}$ & $21,2 \mathrm{ab}$ & $2,8 \mathrm{abc}$ & $2,58 \mathrm{abcd}$ \\
\hline A. Ámbar (T) & 6028 abcde & $65,4 a b c$ & 163 defg & $25,7 \mathrm{jklmn}$ & $\begin{array}{c}15,2 \\
\text { cdefghijklmn }\end{array}$ & $2,8 \mathrm{abc}$ & $2,42 \mathrm{abcd}$ \\
\hline DK-65 (T) & 5763 abcdef & $66,4 \mathrm{a}$ & 157 efghij & $\begin{array}{c}28,0 \\
\text { abcdefghij }\end{array}$ & $\begin{array}{c}14,3 \\
\text { fghijklmn }\end{array}$ & $2,7 \mathrm{abc}$ & $2,65 \mathrm{abcc}$ \\
\hline RB-4000 (T) & 5700 abcdefgh & 63,6 abcdef & 164 defg & 29,7 abcd & $12,2 \mathrm{klmno}$ & $2,7 \mathrm{abc}$ & 2,68 abcd \\
\hline $\begin{array}{l}\text { RB-3006 } \\
(27 x R T x 430)(T)\end{array}$ & $\begin{array}{c}5467 \\
\text { abcdefghij }\end{array}$ & 57,3 nopqr & 154 ghijkl & 25,9 hijklm & $\begin{array}{c}14,7 \\
\text { efghijklmno }\end{array}$ & $2,6 a b c$ & $2,27 \mathrm{~cd}$ \\
\hline RB-27xRTx437 & $\begin{array}{c}5152 \\
\text { abcdefghij }\end{array}$ & 56,6 opqrn & 148 jklmno & $24,1 \mathrm{~nm}$ & $\begin{array}{c}15,9 \\
\text { cdefghijklm }\end{array}$ & $2,7 \mathrm{abc}$ & $2,42 \mathrm{abcd}$ \\
\hline RB-4040 (T) & $\begin{array}{c}4960 \\
\text { abcdefghij }\end{array}$ & 61,3 efghijkl & 139 nopqrs & $\begin{array}{c}28,2 \\
\text { abcdefghij }\end{array}$ & $\begin{array}{c}14,9 \\
\text { cdefghijklmn }\end{array}$ & $2,7 \mathrm{abc}$ & $2,56 \mathrm{abcd}$ \\
\hline $\begin{array}{l}\text { RB-3030 }(5 \times R T \times 430) \\
(\mathrm{T})\end{array}$ & $\begin{array}{c}4951 \\
\text { abcdefghij }\end{array}$ & 57,6 opqrmn & 154 ghijkl & 25,8 ijklmn & 16,5 cdefghi & $2,7 \mathrm{abc}$ & $2,48 \mathrm{abcc}$ \\
\hline DK-47 (T) & $\begin{array}{c}4930 \\
\text { abcdefghij }\end{array}$ & 59,1 ijklmno & 136 qrst & 26,2 fghijklm & $\begin{array}{c}15,3 \\
\text { cdefghijklmn }\end{array}$ & $2,6 \mathrm{abc}$ & $2,37 \mathrm{abcc}$ \\
\hline Pioneer 82G63 (T) & 4796 bcdefghij & $\begin{array}{c}58,5 \\
\text { klmnlopq }\end{array}$ & 142 mnopqr & 28,9 abcde & 11,9 mno & $2,9 a b c$ & $2,86 \mathrm{abcd}$ \\
\hline Pioneer 84G62 (T) & 4256 ghij & $\begin{array}{c}58,8 \\
\text { jklmnopq }\end{array}$ & $126 \mathrm{u}$ & $\begin{array}{c}26,6 \\
\text { efghijklmn }\end{array}$ & 11,4 no & $3,2 \mathrm{abc}$ & $3,03 \mathrm{abc}$ \\
\hline $46038 \times 204$ & 4242 ghij & $56,1 \mathrm{opqr}$ & $130 \mathrm{stu}$ & 25,8 hijklmn & $\begin{array}{c}16,3 \\
\text { cdefghijk }\end{array}$ & $3,4 \mathrm{abc}$ & $3,16 \mathrm{a}$ \\
\hline $\mathrm{Z}-400(\mathrm{~T})$ & 4218 ghij & $\begin{array}{c}58,4 \\
\text { klmnopqr }\end{array}$ & $127 \mathrm{tu}$ & $24,5 \mathrm{mn}$ & $\begin{array}{c}14,1 \\
\text { fghijklmno }\end{array}$ & $2,7 \mathrm{abc}$ & $2,72 \mathrm{abcd}$ \\
\hline RB-46038x RTx437 & 4215 hij & 57,6 opqrmn & $132 \mathrm{rstu}$ & $24,8 \mathrm{mn}$ & 17,3 bcdefgh & $2,9 \mathrm{abc}$ & $2,58 \mathrm{abcc}$ \\
\hline RB-27x204 & $4111 \mathrm{ij}$ & $55,9 \mathrm{pqr}$ & 137 pqrst & $24,2 \mathrm{mn}$ & $\begin{array}{c}14,7 \\
\text { fghijklmno }\end{array}$ & $3,0 \mathrm{abc}$ & $2,60 \mathrm{abcc}$ \\
\hline RB-5x204 & $4064 \mathrm{ij}$ & $55,3 \mathrm{r}$ & $132 \mathrm{rstu}$ & $23,2 \mathrm{n}$ & $\begin{array}{c}14,4 \\
\text { fghijklmno }\end{array}$ & $2,9 \mathrm{abc}$ & $2,80 \mathrm{abcd}$ \\
\hline 46038x RTx435 & $4001 \mathrm{j}$ & 58,3 opqrmnl & $128 \mathrm{tu}$ & $24,4 \mathrm{mn}$ & 17,4 bcdefg & $3,3 a b$ & $3,14 \mathrm{ab}$ \\
\hline Media & 5269 & 60,58 & 152 & 27,26 & 15,25 & 2,75 & 2,52 \\
\hline $\mathrm{CV}$ & 14,57 & 2,64 & 6,66 & 5,64 & 15,06 & 19,13 & 19,29 \\
\hline
\end{tabular}

$\mathrm{REN}=$ Rendimiento de grano $\mathrm{kg} / \mathrm{ha} ; \mathrm{DF}=$ días a floración; $\mathrm{AP}=$ altura de planta en $\mathrm{cm} . \mathrm{LP}=$ longitud de panoja en $\mathrm{cm}$; $\mathrm{EX}=$ longitud de excersión en cm; $\mathrm{CP}=$ calificación de planta; $\mathrm{IEF}=$ incidencia de enfermedades foliares. Estas últimas dos características se calificaron en forma visual de mayor a menor mediante una escala de 1 al 5.

$\mathrm{CV}=$ coeficiente de variación.

Valores para la misma variable respuesta con las mismas letras en forma vertical son considerados iguales de acuerdo con Tukey $(\mathrm{p} \leq 0,05)$. 
Cuadro 6. Comparación de medias mediante la prueba de Tukey $(p \leq 0,05)$ de híbridos y progenitores de sorgo evaluados en Estación Cuauhtémoc, Tamaulipas, México ciclo P-V 2008. Mejores cinco, peores cinco híbridos experimentales.

\begin{tabular}{|c|c|c|c|c|c|c|c|}
\hline Genotipo & REN (kg/ha) & DF & $\mathbf{A P}(\mathbf{c m})$ & $\mathbf{L P}(\mathbf{c m})$ & $\mathbf{E X}(\mathbf{c m})$ & $\mathbf{C P}$ & IEF \\
\hline \multicolumn{8}{|c|}{ Híbridos experimentales } \\
\hline RB-116xRTx437 & $5073 \mathrm{a}$ & 72,8 cdefg & $1,51 \mathrm{abcd}$ & 26,95 bcde & $19,05 \mathrm{abc}$ & 2,13 & 2,05 bcde \\
\hline RB-104xRT x437 & $5034 \mathrm{a}$ & 74,3 bcdefg & 1,49 abcd & 27,75 abcde & 17,40 abcde & 2,13 & 2,13 abcde \\
\hline RB-119xRTx437 & $4987 \mathrm{ab}$ & 75,5 abcde & $1,50 \mathrm{abcd}$ & 27,75 abcde & 11,85 defg & 2,28 & 2,28 abcde \\
\hline RB-116xRTx430 & $4787 \mathrm{abc}$ & 72,5 cdefg & 1,47 abcde & 28,55 abcde & 16,70 abcde & 2,13 & 2,03 bcde \\
\hline RB-118xRTx437 & $4771 \mathrm{abc}$ & 73,8 cdefg & 1,43 bcdefgh & 28,25 abcde & 13,80 cdef & 2,25 & 2,25 abcde \\
\hline RB-106xRT x437 & 4310 abcde & 73,3 cdefg & 1,46 abcdefg & 25,55 cdefg & $19,15 \mathrm{abc}$ & 2,25 & 2,20 abcde \\
\hline RB-119x25 CEA & 4187 abcde & 75,8 abcd & $1,55 \mathrm{ab}$ & $30,15 \mathrm{ab}$ & 11,90 defg & 2,78 & $2,78 \mathrm{abcd}$ \\
\hline RB-118x25 CEA & 4068 abcde & 74,8 bcdef & 1,43 bcdefgh & $29,2 \mathrm{abc}$ & 16,55 abcde & 2,50 & 2,50 abcde \\
\hline RB-106x25 CEA & 3930 abcde & $75,8 \mathrm{abcd}$ & $1,51 \mathrm{abc}$ & 26,4 bcdef & $21,20 \mathrm{ab}$ & 2,58 & 2,53 abcde \\
\hline RB-116x25 CEA & 3716 abcdef & 75,5 abcde & $1,59 \mathrm{a}$ & $30,3 \mathrm{ab}$ & 15,60 bcdef & 2,65 & 2,65 abcde \\
\hline Medias experimentales & 4467 & 74,5 & 1,48 & 28,1 & 16,1 & 2,40 & 2,35 \\
\hline \multicolumn{8}{|c|}{ Progenitores } \\
\hline RTx437 & 3598 abcdef & 74,0 bcdefg & $1,18 \mathrm{~lm}$ & $22,45 \mathrm{fgh}$ & $14,65 \mathrm{bcdf}$ & 2,00 & $1,88 \mathrm{de}$ \\
\hline LRB-25 CEA & 3502 bcdefg & 74,5 bcdef & 1,35 efghij & 25,50 cdefg & $20,80 a b c$ & 2,83 & $2,83 \mathrm{abc}$ \\
\hline LRB-104B & 3138 defgh & 76,0 abcd & 1,32 hijk & 28,80 abcde & 9,10 fghi & 2,63 & 2,63 abcde \\
\hline LRB-106B & 3128 defgh & $79,3 \mathrm{ab}$ & $1,25 \mathrm{jkl}$ & $22,0 \mathrm{gh}$ & 14,80 bcdef & 2,93 & $2,93 \mathrm{ab}$ \\
\hline LRB-118B & 2812 efgh & $77,0 \mathrm{abc}$ & $1,22 \mathrm{klm}$ & $29,35 \mathrm{abc}$ & $5,85 \mathrm{ghi}$ & 2,70 & 2,70 abcde \\
\hline LRB-119B & $2298 \mathrm{fgh}$ & 80,8 a & 1,30 hijkl & 27,30 abcde & 3,85 hi & 2,70 & 2,70 abcde \\
\hline LRB-116B & $1989 \mathrm{gh}$ & $77,8 \mathrm{abc}$ & 1,37 dfghij & 28,75 abcde & 9,05 fghi & 2,70 & 2,58 abcde \\
\hline $\mathrm{RTx} 430$ & $1804 \mathrm{~h}$ & 76,0 abcd & $1,08 \mathrm{~m}$ & $29,35 a b c$ & $3,45 \mathrm{i}$ & 2,08 & $1,88 \mathrm{de}$ \\
\hline Medias proge. & 2558 & 76,9 & 1,26 & 26,7 & 10,2 & 2,57 & 2,52 \\
\hline Media general & 3977 & 73,89 & 1,39 & 27,21 & 14,78 & 2,46 & 2,41 \\
\hline $\mathrm{CV}$ & 14,24 & 2,58 & 3,57 & 5,72 & 17,40 & 14,01 & 14,20 \\
\hline
\end{tabular}

$\mathrm{REN}=$ rendimiento de grano $\mathrm{kg} / \mathrm{ha}$; $\mathrm{DF}=$ días a floración; $\mathrm{AP}=$ altura de planta en $\mathrm{cm} ; \mathrm{LP}=$ longitud de panoja en $\mathrm{cm}$; $\mathrm{EX}=$ longitud de excersión en cm; $\mathrm{CP}=$ calificación de planta; $\mathrm{IEF}$ = incidencia de enfermedades foliares. Estas últimas dos características se calificaron en forma visual de mayor a menor mediante una escala de 1 al 5.

$\mathrm{CV}=$ coeficiente de variación.

Valores para la misma variable respuesta con las mismas letras en forma vertical son considerados iguales de acuerdo con Tukey $(\mathrm{p}=0,05)$.

los híbridos experimentales RB-104xRTx437 y RB-27xRTx437 (Montes et al., 2014a; Montes et al., 2014b). Se patentaron respectivamente con los nombres comerciales de RB-Huasteco y RB-Norteño. La producción comercial de semilla se realiza en Río Bravo, Tamaulipas. Distribuye comercialmente la semilla para su siembra en los estados de Tamaulipas, Nuevo León, Veracruz y Guanajuato. El contar con semilla de híbridos mexicanos, producidos en el país, presenta la ventaja de que los empleos de producción se generan en México y se disminuye la fuga de divisas.
La dependencia de México por semillas importada es grave y si se agrega que también es deficitario en producción de alimentos (45\%), el problema es mayor.

Por otro lado, se encontró una correlación significativa $(p \leq 0,01)$ de $r=0,67$ y $r=0,53$ para rendimiento de grano $\mathrm{kg} / \mathrm{ha}$ y altura de planta respectivamente para todos los experimentos establecidos en este estudio. Esto indica que los híbridos de mayor altura de planta fueron los que presentaron los más altos rendimientos. Esto coincide con lo reportado por Bakeith (1990), Ezeaku 
y Mohammed (2006), Sarvari y Behesthi (2012). Doget (1967) indica que los sorgos de mayor altura de planta tienden a producir mayor rendimiento de grano, debido a que su área fotosintética es mayor. Para obtener correlaciones positivas entre estas dos características, es indispensable contar con amplia variabilidad genética en los materiales utilizados.

En la región de las Huastecas la actividad ganadera es muy importante y durante la época de estiaje (noviembre a mayo) se presenta escasez de forraje. $\mathrm{Al}$ respecto, los resultados indican que puede ser útil la selección de híbridos de mayor altura de planta y apropiados para utilizarse para el aprovechamiento del grano y forraje. Los híbridos experimentales que han comprobado tener potencial de producción de grano para las Huastecas, igualmente pueden tener adaptación para regiones de climas similares $\left(\mathrm{Aw}_{0}\right)$ en México, presentes en partes de los estados de Morelos, Michoacán, Sinaloa, Colima y Nayarit.

\section{Otros caracteres agronómicos}

El análisis de varianza por ambiente para las características estudiadas DF, AP, LP, EX, CP e IEF indicó diferencias significativas $(p \leq 0,01)$ entre genotipos en los tres ambientes y el combinado. Como se mencionó anteriormente, esto es consecuencia de las diferencias genéticas entre los genotipos evaluados. Se detectaron también diferencias altamente significativas $(\mathrm{p} \leq 0,01)$ entre ambientes y la interacción genotipoambiente. Esto significa que el ambiente juega un papel importante en la manifestación de las características en los genotipos, aunque estadísticamente el peso de la interacción fue menor al efecto del genotipo y el ambiente, incluso para el rendimiento de grano (Cuadro 2).

Días a floración. House (1985) reporta valores para la floración en sorgo que varían entre 52,4 a 90,5 días. En este estudio los valores fluctuaron entre 55 a 66,4 días, por lo que los valores encontrados se ubican más bien dentro de los genotipos precoces. Los híbridos experimentales que resultaron más precoces se relacionan con que en su formación intervienen líneas progenitoras masculinas precoces como 204 y femeninos como 46038, 64, 5 y 27. Los sorgos de ciclo precoz presentan ventajas cuando la estación de crecimiento es corta y cuando se presentan periodos de sequía y altas temperaturas (Murphy et al., 2014). Las condiciones climáticas que se presentan en la región de las Huastecas es irregular, por lo que es frecuente que se presenten condiciones de sequía y altas temperaturas. El mejor híbrido experimental RB-104xRTx437 presentó una ventaja adicional en la floración, ya que $(\mathrm{p} \leq 0,05)$ fue más precoz $(62)$ que A. Ámbar (65) y DK-65 (66).

Altura de planta. Los híbridos más altos fueron los experimentales RB-5x430 REA $(198 \mathrm{~cm})$ y RB27x430 REA $(200 \mathrm{~cm})$. Los sorgos con alturas de planta superiores a $1,70 \mathrm{~m}$ son indeseables, ya que suelen presentar problemas de acame y dificultad para la cosecha mecánica. La mayor altura de planta de estos híbridos se asocia al progenitor masculino 430 REA.

Longitud de panoja. También se observó que los híbridos experimentales que presentaron los más altos rendimientos, p.e RB-Patrón, fueron los que tuvieron la mayor longitud de panoja. Castro et al. (2000) encontraron que los genotipos clasificados como resistentes a sequía, produjeron más grano debido a una mayor longitud de la panoja. El número de granos y el peso por panoja son los componentes de rendimiento más importantes (Ezeaku y Mohammed, 2006). El carácter longitud de exerción presentó una variación de 10,8 a 21,8 cm. House (1985) indica que los sorgos con buena exerción son aquellos que presentan más de $10 \mathrm{~cm}$; por lo tanto, todos los genotipos presentan una exerción adecuada para la cosecha mecánica del grano.

Enfermedades foliares. La enfermedad foliar que se presentó con mayor incidencia $(80 \%)$, fue el tizón de la hoja (Helmintosporium turcicum (Pass.) Leo and Suggs). En menor grado se presentaron antracnosis (Colletotrichum graminicola (Ces.) Wilson) y mancha zonada (Gleocercospora sorghi D. Bain y Edg). Se encontró correlación $(\mathrm{p} \leq 0,01)$ entre incidencia de enfermedades foliares y calificación de planta de $\mathrm{r}=0,81$ y 0,94 respectivamente para todos los experimentos realizados. Los resultados variaron entre 2,4 a 3,4. La presencia de enfermedades foliares en el cultivo de sorgo (S. bicolor) es un problema importante en el sur de Tamaulipas (Williams, 2002; Díaz y Montes, 2008). $\mathrm{El}$ ataque de este tipo de enfermedades pueden resultar en la defoliación prematura de la planta, la reducción del contenido de azúcar en el tallo y la disminución del rendimiento de grano (Girad, 1980). 


\section{Comparación de híbridos y progenitores}

Al comparar los híbridos experimentales con sus progenitores se observó que los híbridos presentaron una altura de planta superior a sus padres (Cuadro 6), lo cual coincide con lo reportado por Kirby y Atkins (2000). House (1985) reporta la presencia de genes dominantes para altura de planta mayor, siendo esta una explicación razonable del valor mayor de altura de planta de los híbridos. También fueron más precoces igual a lo reportado por Mahdy et al. (2011). Además se observó que fueron de mayor longitud de la espiga y excersión y tuvieron menor incidencia de enfermedades foliares. En promedio, se encontró una heterosis de 59,9, -2,3, 17,8, 7,0, 57,5, $-5,6$ y $-5,0 \%$ respectivamente para REN, DF, AP, LP, EX. CP e IEF (datos no presentados). En las mismas características se encontró una heterobeltiosis de 46,1, $-4,9,12,6,1,6,18,7,-14,1$ y $-14,6$ respectivamente. Esto es coincidente con lo encontrado por Kirby y Atkins (2000), Mahdy et al. (2011), Kenga et al. (2003) y Cisneros et al. (2007). En promedio, en los híbridos experimentales, se presentó una heterosis de $59,9 \%$ y heterobeltiosis de $49,1 \%$ lo cual fue alto. Los híbridos con mayor heterosis y heterobeltiosis para rendimiento de grano fueron: RB-116xRTx430 con $152,5 \%$ y $146,7 \%$ y RB-119xRTx437 con 117,4 y $94 \%$ respectivamente. Estos dos híbridos se encuentran en el grupo superior para rendimiento de grano. El mejor híbrido RB-104xRTx437, presentó una heterosis para rendimiento de grano de $49,5 \%$ y una heterobeltiosis de $39,5 \%$. Esto se debe al mayor rendimiento de grano presente en el progenitor masculino (RTx437), lo cual incide en el cálculo de los valores obtenidos. Para días a floración, calificación de planta (CP) e incidencia de enfermedades foliares (IEF), se presentó una heterosis y heterobeltiosis negativa. Esto concuerda con lo reportado por Mahdy et al. (2011) para días a floración. Se comprueban las hipótesis planteadas en este trabajo: los híbridos experimentales fueron iguales a los mejores comerciales y fueron diferentes a sus padres.

El hecho de que los híbridos resulten más precoces que sus padres no representa ningún problema agronómico, ya que es una característica deseable. Respecto a enfermedades foliares se complica la selección de híbridos tolerantes, ya que ambos progenitores deben presentar buen grado de tolerancia.

\section{LITERATURA CITADA}

Assefa, Y., y S.A. Staggenborgh. 2009. Grain yield with hybrid advancement and changes in agronomic practices from 1957 through 2008. Agron. J. 102:703-706.

Bakheith, R.B. 1990. Variability and correlations in grain sorghum genotypes (Sorghum bicolor [L.] Moench) under drought conditions at different stages of growth. Crop Sci. 185:355-360.

Castro, S.N., C.J. Ortiz, M.M.D.C. Castillo, y G.F. Zavala. 2000. Producción de biomasa en líneas de sorgo como respuesta al estrés hídrico. Rev. Fitotec. Mex. 23:321-334.

Cisneros, M.E., L.E. Mendoza, G. Mora, L. Córdoba, y M. Livera. 2007. Híbridos y progenitores de sorgo tolerantes al frío. II: Efecto de Fusarium verticilloides (Sacc) Nirenberg en el rendimiento de semilla y sus componentes en condiciones de campo. Agrociencia 41:283-294.

CONACYT. 2013. Fondo sectorial de investigación en materia agrícola, pecuaria, acuacultura. Convocatoria 20132. http://www.conacyt.gob.mx/index.php/el-conacyt/ convocatorias-y-resultados-conacyt/convocatoriasfondos-sectoriales-constituidos/convocatoriasagarpa-conacyt/convocatorias-cerradas-sagarpaconacyt/3903--445/file (Consultado 14 agosto 2014).

Díaz, F.A., y G.N.B. Montes. 2008. La fitopatología en la región semiárida de Tamaulipas, México: reseña histórica. Rev. Mex. Fitopatol. 26:62-70.

Doget, N. 1967. Yield increase from sorghum hybrids. Nature 216:798-799.

Duvick, D.N. 1999. Heterosis: feeding people and protecting natural resources. En: J. G. Coors, y S. Pandey, editores, The genetics and exploitation of heterosis in crops. American Society of Agronomy, Inc., Crop Science of America, Inc., Soil Science Society of America, Inc., Madison, Wi, USA. p. 19-29.

Ezeaku, I.E., y S.G. Mohammed. 2006. Character association and path analysis in grain sorghum. Afr. J. Biotechnol. 5:1337-1340.

Financiera Rural. 2011a. Monografía del sorgo grano. http:// www.financierarural.gob.mx/informacionsectorrural (Consultado 23 nov. 2013).

Financiera Rural. 2011b. Semillas para siembra de maíz, trigo, sorgo y frijol. http://www.financierarural.gob. $\mathrm{mx} /$ informacionsectorrural/Documents/Monografias/ Monograf\%C3\%ADaSemillas\%28oct11\%29vf.pdf (Consultado 14 agosto 2014).

Flores-Naveda, A., C.G.S. Valdés-Lozano, F. Zavala-García, E. Olivares-Sáenz, A. Gutiérrez-Díe, y M.E. VázquezBadillo. 2013. Comportamiento agronómico de 
líneas para la producción de semilla de sorgo. Agron. Mesoam. 24:119-131

García, E. 1988. Modificaciones al sistema de clasificación climática de Koppen. Universidad Nacional Autónoma de México. México, D.F., México.

Girard, J.C. 1980. A review of souty stripe and rouge, zonate and oval leaf spots. En: R.J. Williams, R.A. Frederiksen, y L.K. Mughogho, editores, Sorghum diseases, a world review. International Crops Research Institute for the Semi-Arid Tropic ICRISAT, Patancheru (AP). India. p. 229-239.

House, L.R. 1985. A guide to sorghum breeding. Ed. ICRISAT. Andhra Pradesh, India.

INIFAP (Instituto Nacional de Investigaciones Forestales, Agrícolas y Pecuarias). 2013. Campo Experimental Huastecas. http://www.inifapcirne.gob.mx/ Localizacion.htm (Consultado 9 mar. 2014).

Kenga, R., S.O. Alabi, y S.Y. Grupta. 2003. Yield stability of sorghum hybrids and parental lines. Afr. Crop Sci. J. 11:65-73.

Kirby, J.S., y R.E. Atkins. 2000. Heterotic response for vegetative mature plant characters in grain sorghum Sorghum bicolor (L.) Moench. Crop Sci. 40:319-329.

León-Velasco, H., L.E. Mendoza-Onofre, F. CastilloGonzález, T. Cervantes-Santana, y A. MartínezGarza. 2009. Evaluación de dos generaciones de híbridos y progenitores de sorgo tolerantes al frio. II: Aptitud combinatoria, heterosis y heterobeltiosis. Agrociencia 43:609-623.

Mahdy, E.E., M.A. Ali, y A.M. Mahmoud. 2011. The effect of environment on combining ability and heterosis in grain sorghum (Sorghum bicolor L. Moench). Asian J. Crop Sci. 3:1-15.

Montes, G.N., A.H. Williams, C.G. Arcos, Q.V. Pecina, C.M. De la Garza, G.T. Moreno, y V.E. Vargas. 2014a. RBHuasteco, sorgo de grano para áreas de riego y buen temporal. Revista Mexicana de Ciencias Agrícolas. Pub. Esp. No. 7. 1331-1335.

Montes, G.N., A.H. Williams, C.G. Arcos, V.E. Vargas, Q.V. Pecina, y M.R. Espinosa. 2014b. RB-Norteño, sorgo de grano para áreas con sequía. Revista Mexicana de Ciencias Agrícolas. Pub. Esp. No. 7. 1337-1342.

Murphy, R.L., D.T. Morishige, J.A. Brady, W.L. Rooney, S. Yang, P.E. Klein, y J.E. Mullet. 2014. Ghd7 (Ma6) represses sorghum flowering in long days: Ghd7 alleles enhance biomass accumulation and grain production. The Plant Genome 7:1-10.
Reddy, B.V.S., A.A. Kumar, y W.D. Dar. 2006. Overview of sweet sorghum breeding at ICRISAT: Opportunities and constraints. http://www.ifad.org/events/ sorghum/b/Reddy.pdf (Cosultado 20 dic. 2013).

Reddy, B.V.S., A.A. Kumar, y W.D. Dar. 2006. Overview of sweet sorghum breeding at ICRISAT: Opportunities and constraints. www.ifad.org/events/sorghum/b/ Reddy.pdf (Cosultado 20 diciembre 2013).

Rooney, W.L., F.R. Miller, y L.W. Rooney. 2003. Registration of RTx437 sorghum parental line. Crop Sci. 43:445-446.

Sarvari, S.M., y S.A. Behesthi. 2012. Relationship between grain yield and plant characteristics in grain sorghum genotypes under drought stress conditions. Iranian J. Crop Sci. 14:183-201.

SAS Institute. 1999. User's guide. SAS Sofware Release 8.0. Cary, NC, USA.

SIAP (Servicio de Información Agroalimentaria y Pesquera). 2011. http://reportes.siap.gob.mx/agricola_siap/ cultivo/index.jsp. Consultado 17 nov. 2011.

Stephens, J.C., y R.F. Holland. 1954. Cytoplasmic male sterility for hybrids sorghum seed production. Agron. J. 46:20-23.

Williams, A.H. 1981. Dos nuevos sorgos híbridos de grano para el norte de de Tamaulipas INIA RB-3030 e INIA RB-3006. Campo Agrícola Experimental Rio Bravo. Folleto técnico No. 2. Instituto Nacional de Investigaciones Agrícolas. Río Bravo, Tamaulipas, México.

Wllliams, H. 1988. RB-4000 híbrido de sorgo para áreas de riego. Campo Agrícola Experimental Río Bravo. Folleto técnico No. 8. Instituto Nacional de Investigaciones Agrícolas. Rio Bravo, Tamaulipas, México.

Williams, H. 1996. RB-4040 nuevo híbrido de sorgo para el noreste de México y tolerante a Sporisorium reilianum y Macrophomina phaseolina. Rev. Fitotec. Mex. 19:193-194.

Williams, H. 2002. Sorghum and millet in Mexico. En: J.F. Leslie, editor, Sorghum and millet diseases. Iowa State Press, Ames, USA. p. 457-463.

Williams, H., N. Montes, y V. Pecina. 2006. Sorgo. En: L.A. Rodríguez del Bosque, editor, 50 años de investigación agropecuaria en el norte de Tamaulipas, historia, logros y retos. Libro técnico No. 1. Campo Experimental Río Bravo, Instituto Nacional de investigaciones Forestales Agrícolas y Pecuarias. Río Bravo, Tam., México. p. 33-54. 
Williams, H., R. Rodríguez-Herrera, y N. Montes-García. 1995. 20 años de investigación en sorgo en el Campo Experimental Río Bravo. Germen 11:1-35

Williams, H., V. Pecina, F. Zavala, y N. Montes. 2004. RB-Patrón, nuevo híbrido de sorgo para grano en el noreste de México. Rev. Fitotec. Mex. 27:291-293.

Williams, H., V. Pecina, N. Montes, G. Arcos, F. Zavala, y V.A.J. Gámez. 2009a. Incidencia de carbón de la panoja Sporisorium reilianum (Kühn) Langdon y
Fullerton en híbridos de sorgo [Sorghum bicolor (L.) Moench.] para grano. Rev. Mex. Fitopatol. 27:36-44.

Williams, H., V. Pecina, N. Montes, F. Zavala, G. Arcos, y A.J. Gámez. 2009b. Evaluación de híbridos de sorgo Sorghum bicolor (L.) Moench para resistencia a pudrición carbonosa del tallo Macrophomina phaseolina (Tassi) Goid en Tamaulipas, México. Rev. Mex. Fitopatol. 27:69-72. 
